\title{
The SENSE Project: Developments, Characteristics and Application of Low Light-Level Photosensors
}

\author{
Thomas Huber $^{* 1,2}$, Thomas Berghöfer ${ }^{3}$, Domenico della Volpe ${ }^{4}$, Andreas Haungs ${ }^{1}$, \\ Katharina Henjes-Kunst ${ }^{3}$, Katrin Link ${ }^{1}$, Razmik Mirzoyan ${ }^{5}$, Teresa Montaruli ${ }^{4}$, \\ Andrii Nagai ${ }^{4}$, Derek Strom ${ }^{5}$ - the SENSE Consortium \\ ${ }^{1}$ Institut für Kernphysik, Karlsruhe Institute of Technology, Karlsruhe, Germany \\ ${ }^{2}$ DESY in Zeuthen, Germany \\ ${ }^{3}$ DESY in Hamburg, Germany \\ ${ }^{4}$ Dep. de physique nuclaire et corpusculaire, Université de Geneve, Switzerland \\ ${ }^{5}$ Max Planck Institute for Physics, Munich, Germany \\ E-mail: thomas.huberakit.edu
}

\begin{abstract}
The SENSE project is a Horizon 2020 Coordination and Support Action aiming to coordinate research and development efforts in academia and industry in low light-level sensoring. In addition, SENSE aims to transfer knowledge by initiating information and training events and material, for sharing the status of existing LLL sensors and providing information by outreach experiments and activities. This contribution focuses on developments, characteristics and existing calibration setups within the SENSE partner institutions for photo-sensing technologies like Silicon Photomultiplier (SiPM). A short summary of the SENSE roadmap for the development of low lightlevel sensors will be given. In addition, a specific example of application of SiPMs in the field of astroparticle physics experiments will be discussed in more details, namely the scintillation detector readout for the surface detector enhancement of the IceCube observatory.
\end{abstract}

36th International Cosmic Ray Conference -ICRC2019-

July 24th - August 1st, 2019

Madison, WI, U.S.A.

\footnotetext{
* Speaker.
} 


\section{Introduction}

The aim of SENSE is a coordination of European research groups concerned with low light-level sensors. Representatives from academia and industry pointed out that developments could be made faster when one or a small number of labs could take the lead of these activities and work in close collaboration with industry. This advice is considered in SENSE. By formulating a roadmap incorporating the $R \& D$ activities necessary for the development of the ultimate low light-level sensors, the $R \& D$ efforts of European research groups, industry, and strategic partners worldwide are efficiently aligned. The project joins forces of European experts in developing the ultimate low light-level sensors and taking the leadership in R\&D activities. Following new and emerging technologies in detecting minimal quantities

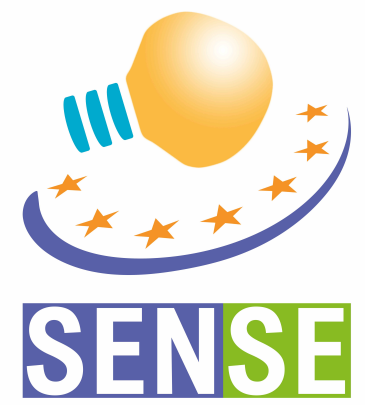

Figure 1: Logo of the SENSE Project. of light (single photons) is a challenge. Common, coordinated measurements of SiPM characteristics are performed. SiPMs from different companies, measured at different labs, are compared and the results are published in [1], [2] and [?]. The joint efforts are regulated in a Cooperation agreement, signed by nine institutes.

SENSE further aims at attracting and supporting young researchers to get involved in forefront technology developments. This was realized by well-defined technology sessions at diverse summer schools and a SENSE Detector School. A dedicated Training and Learning section will be available at the SENSE website (www.sense-pro.org). The website constitutes the main tool for dissemination and exploitation of results to a broader public. It details the goals, status, and results of the project and provides information about photosensors as well as test facilities and experiments using photosensors. Furthermore, it lists upcoming events interesting for the community.

\section{The SENSE Roadmap}

The SENSE roadmap aims to define the R\&D activities that the SENSE Project is following towards the development of the ultimate low light-level (LLL) sensor(s), mainly for future astroparticle physics projects, but also medical, automotive, biology, and safety applications. We focus on developments that are crucial for two photo-sensing technologies; silicon photomultipliers (SiPMs) and photomultipliers (PMTs). We have identified three major sectors of development for each technology: (1) the performance of the sensors (which typically depends on the application), (2) the readout and control electronics, and (3) the integration of the electronics into the sensor. For each sector, we point out the specifications required to address individual fields of application and the challenges which must be overcome. The final roadmap including specific recommendations will be published at the end of the project in 2019 .

\subsection{The State of the Art}

All innovation with respect to LLL sensors is driven by the challenging demands by research projects and infrastructures. Medical diagnostic instrumentation is one of the largest consumer 

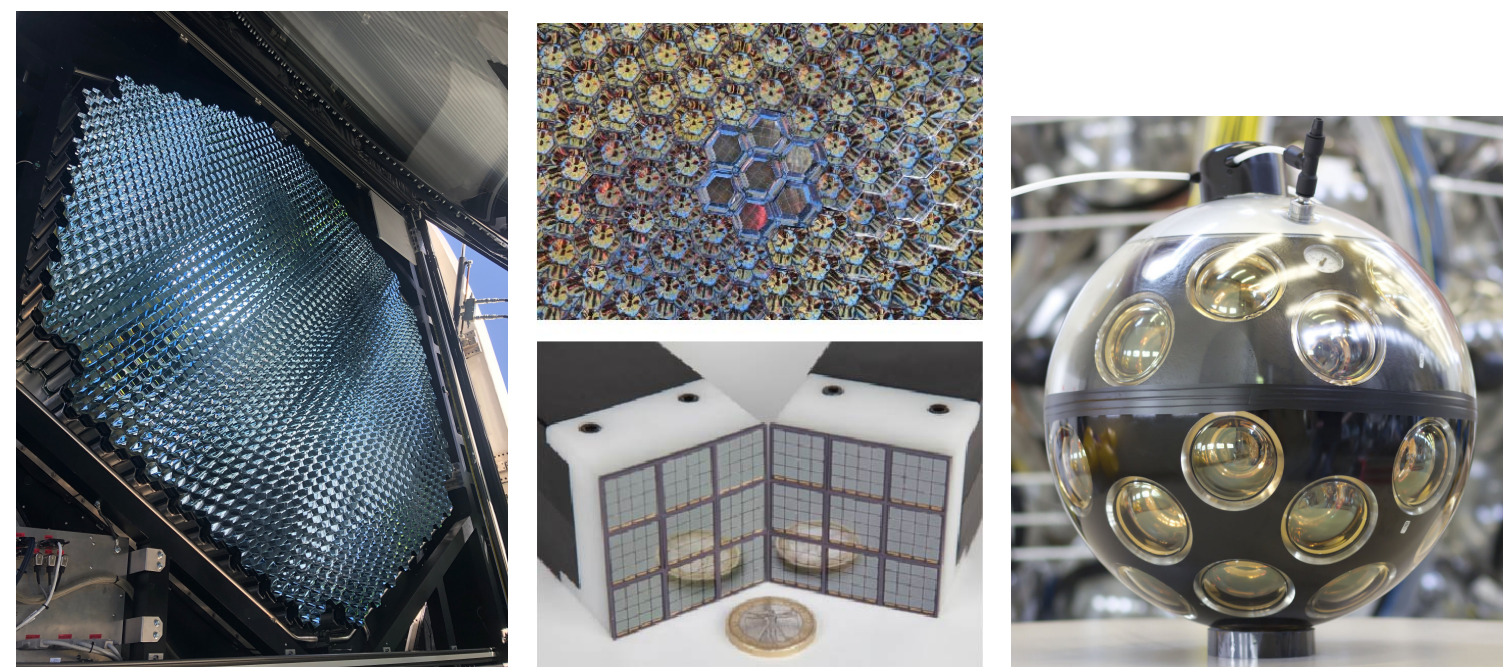

Figure 2: Left: Picture of the LST Camera. Photo credit: Takayuki Saito. Top center: Picture of a SiPM based cluster installed in the MAGIC telescope camera system. Photo credit: Alexander Hahn. Bottom center: Picture of SiPM matrices. Right: Pictures of PMTs used in a KM3NeT DOM.

of PMTs, with about 600,000 PMTs/year, where they are used in Positron Emission Tomography (PET), in gamma-ray cameras, and in many applications in the life sciences. Besides specific applications of PMTs, e.g. in the oil drilling industry, large-scale experiments in basic research are consumers of several tens of thousands LLL sensors, albeit the net consumption varies from year to year. An astroparticle physics experiment, such as the Cherenkov Telescope Array (CTA) [4], will use on the order of 200,000 SiPMs and PMTs in the telescope cameras. KM3NeT [5] will be composed of 3 building blocks with 115 strings comprising 18 optical modules of 17 -inch diameter, each of them composed of 31 PMTs of 3-inch diameter. The total number of PMTs comprising KM3Net will be of the same order as CTA. The demand of astroparticle, particle, and nuclear physics experiments to reach an ever higher level of precision in light detection, with broader dynamic range going from 1 to thousands of photons and with high efficiency is one of the main $R \& D$ drivers in the domain of the LLL detection.

The market for LLL sensors in the context of future upgrades of astroparticle projects is huge. It was estimated in 2010 that approximately 0.5 Billion Euro should be spent in the next decade on photosensors. SENSE is currently working to examine the photosensor developments for existing infrastructures, their major upgrades and upcoming projects. It is also examining products from industry and their performance. The work (within the SENSE project) was discussed during the SENSE TechForum on photosensing and associated electronics on June 21-22, 2018 in Geneva. In the following subsections we introduce the two main photosensor types (SiPMs and PMTs) currently used for LLL detection in scientific experiments and provide a few examples of other types of photosensors.

\subsection{SiPMs}

SiPM technology was invented in 1993 by Boris Dolgoshein (MEPhI) and first used in particle physics experiments such as T2K [6], and the first mass implementation was carried out in DESY 
with MEPHI/Pulsar SiPMs (7.6K devices) in 2003 [7]. It was then followed by several astroparticle physics experiments (e.g. in very high energy imaging Cherenkov telescope gamma-ray cameras, in the double beta decay experiment GERDA [8], for readout of scintillator detectors, and in dark matter experiments). The GERDA experiment uses a combination of PMTs and SiPM. It is the first experiment with a large SiPM array operated at cryogenic temperature.

The first application to ground-based gamma-ray astrophysics is the FACT camera [9], followed by the imaging cameras of the three different Small Size Telescopes (SST) of the CTA collaboration (SST-1M, ASTRI, GCT) and of the Schwarzschild-Couder Middle Size Telescope (MST). Additionally, a SiPM-based sensor clusters for the MAGIC telescope project [10] is under extensive tests and evaluation. First prototypes are in the test phase for SiPM-based fluorescence cameras (EUSO-Balloon [11], FAMOUS [12] at Auger) and for light detection in future Dark Matter experiments (DarkSide [13], DARWIN [14]).

\begin{tabular}{|l|c|c|}
\hline & $\mathbf{2 0 1 0}$ & $\mathbf{2 0 1 5}$ \\
\hline PMT & & $36-43 \%$ \\
\hline $\begin{array}{l}\text { Peak Quantum Effi- } \\
\text { ciency (QE) }\end{array}$ & $28-34 \%$ & $94-98 \%$ \\
\hline $\begin{array}{l}\text { Photoelectron Col- } \\
\text { lection Efficiency on } \\
\text { the } 1^{\text {st }} \text { Dynode }\end{array}$ & $60-80 \%$ & $<0.02 \%$ \\
\hline $\begin{array}{l}\text { Afterpulse Rate (for } \\
\text { a set threshold } \geq 4 \\
\text { ph.e.s) }\end{array}$ & $0.5 \%$ & $50-60 \%$ \\
\hline SiPM Pak- & $20-30 \%$ & $<2 \%$ \\
\hline $\begin{array}{l}\text { Peak Photon Dection Efficiency } \\
\text { (ect) } \\
\text { (PDE) }\end{array}$ & $30-40 \%$ & $50-100 \mathrm{kHz} / \mathrm{mm}^{2}$ \\
\hline Afterpulse Rate & $>40-60 \%$ & $5-10 \%$ \\
\hline $\begin{array}{l}\text { Dark Count Rate } \\
\text { (DCR) }\end{array}$ & $1-3 \mathrm{MHz} / \mathrm{mm}^{2}$ & 50 \\
\hline Crosstalk & & \\
\hline
\end{tabular}

Currently, a large variety of SiPM matrices are available on the sensor market. There also exists a variety of alternative commercial readout solutions. SiPM matrices with improved filling factor are currently being developed to overcome the low geometrical efficiency of these devices. However, for fast timing applications the size of SiPMs is limited to several $\mathrm{mm}$, due to the charge collection time. Furthermore, an increased cell size would unfortunately increase both its gain and undesired crosstalk.

SiPM-based matrices with complete readout, as in a CMOS (or CCD) camera, will be scalable and would allow a simple assembly in arbitrary shapes, arriving to large coordinate-sensitive imaging cam-

eras. However, stray heat might cause a problem in fast on-chip digital readout solutions.

Depending on the wavelength, radiation background, temperature, cost, and application, SiPM solutions have comparable photon-detection efficiencies to PMTs, albeit for the moment PMTs are chosen to cover surface areas larger than $1 \mathrm{~m}^{2}$.

This is mostly due to the price and the reduced number of readout channels. The work of SENSE addresses this issue as well as the signal-to-noise ratio, which for comparable temperatures and acquisition thresholds below 1 photoelectron (ph.e.) is still better for PMTs. Surely, SiPMs are very desirable for most applications, since they are more robust and require lower voltage.

Table 1 provides a comparison of basic parameters for PMTs and SiPMs available in 2010 and 2015, where the improvements of both technologies are clearly demonstrated. 


\subsection{PMTs}

PMTs are produced by companies in various sizes, from very small $(\sim 1 \mathrm{~cm}$ in size) to very large (up to $50 \mathrm{~cm}$ in diameter). PMTs selected from Hamamatsu for the CTA project are now confirming the expected high quality performance of these devices. Measurements show substantially better performance of these devices than the requirements for parameters such as QE, afterpulse rates, and Peak/Valley ratio of single photon counting set by the CTA collaboration. ETEL and Hamamatsu provide the PMTs to KM3NeT.

Cryogenic PMTs are currently the standard light sensors applied in dark matter searches using liquid Argon and Xenon (LAr and LXe, respectively). In addition to the standard requirements, such as high QE, low DCR and stable performance, these PMTs also need to be optimized for very low radioactive contamination. Low Uranium and Thorium content is necessary to suppress any neutron background.

Despite the large request, the number of companies worldwide producing PMTs $(\leq 5)$ is relatively small, which impairs fruitful competition.

\subsection{Other Sensors}

Gaseous PMTs (GPMs) are explored as an alternative in LLL detection in cryogenic applications. This technology may provide a high filling factor and allow to fully surround an experiment and to detect light in all directions. First measurements with 4-inch GPMs demonstrate a large dynamic range, good stability, energy and time resolution, as well as a low DCR.

The ABALONE Photosensor Technology is a modern, cost-effective, and uniquely robust technology that provides sensitivity to visible and UV light, exceptional radio-purity, low afterpulsing noise, and high overall detection performance. Therefore, it can open new horizons in all application areas that require robust and modular large-area photosensors, including large experiments in fundamental science, as well as new types of scanners for both functional medical imaging and nuclear security.

The successful application of a tungsten transition-edge sensor (TES) operated below $100 \mathrm{mK}$ in the Any Light Particle Search-II (ALPS-II) experiment [15], to detect single photons in the near-infrared, demonstrates that this technology is currently entering astroparticle physics. One can speculate that further R\&D may help this promising low-background single-photon detection technology to find wider application in research. CRESST-II [16], a direct dark matter search detector, uses scintillating calorimeters to detect nuclear recoils in $\mathrm{CaWO}_{4}$ single crystals. The absorbers are equipped with TES with transition temperature of $15 \mathrm{mK}$ measuring the temperature rise of the crystals due to particle interactions.

\section{SiPM Characterization}

SENSE fostered a cooperation agreement for providing a framework for the testing and performance studies of photosensors. Nine institutions have signed the agreement in carrying out together a long-term detector R\&D for future ultimate LLL photosensors, which also includes an educational and technology training component (see also www.sense-pro.org). An example of these facilities is the measurement setup of KIT, Germany, shortly described in the following. 

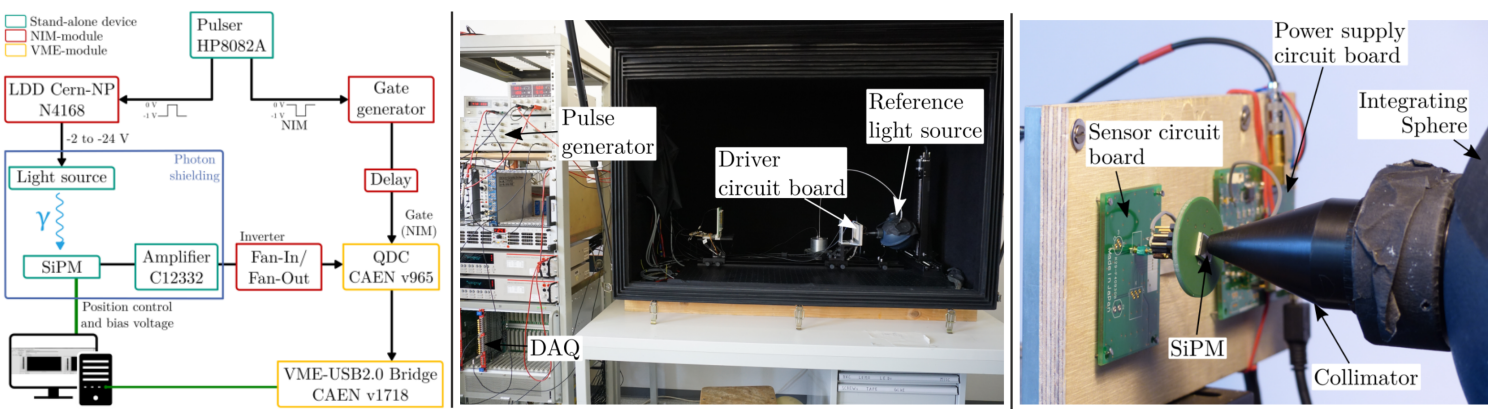

Figure 3: Left: Schematic of SPOCK. Center: Picture of the SPOCK setup. Right: Calibration process of the SiPMs used for the IceCube scintillators described in Chapter 4.

For calibration and sorting tasks of photo detectors, a Single PhOton Calibration stand at KIT (SPOCK) was built. It consists of a reference light source, the sensor to be tested, photon shielding and readout electronics. A schematic view of SPOCK can be seen in Figure 3. The UV-light from the LED-array is diffusely reflected inside the integrating sphere. The sphere's inside is made of PTFE4 which reflects UV-light in the region of 300 to $430 \mathrm{~nm}$. The integrating sphere works as a beam splitter and a diffuser.

The gain of a SiPM channel can be derived easily from the charge spectrum (Figure 4) by determining the distance between consecutive photo electron equivalent peaks (p.e.) [17]. The photo detection efficiency (PDE) is defined as the ratio $P D E=\frac{N_{p e}}{N}$ whereby $N$ is the total number of incident photons to the photosensor and $N_{p e}$ the amount of detected photons and is therefore one of the key benchmarks for a photosensor. For SiPMs, $N_{p e}$ can be obtained by recording the dark count spectrum and the single photon spectrum (Figure 4) of the device.
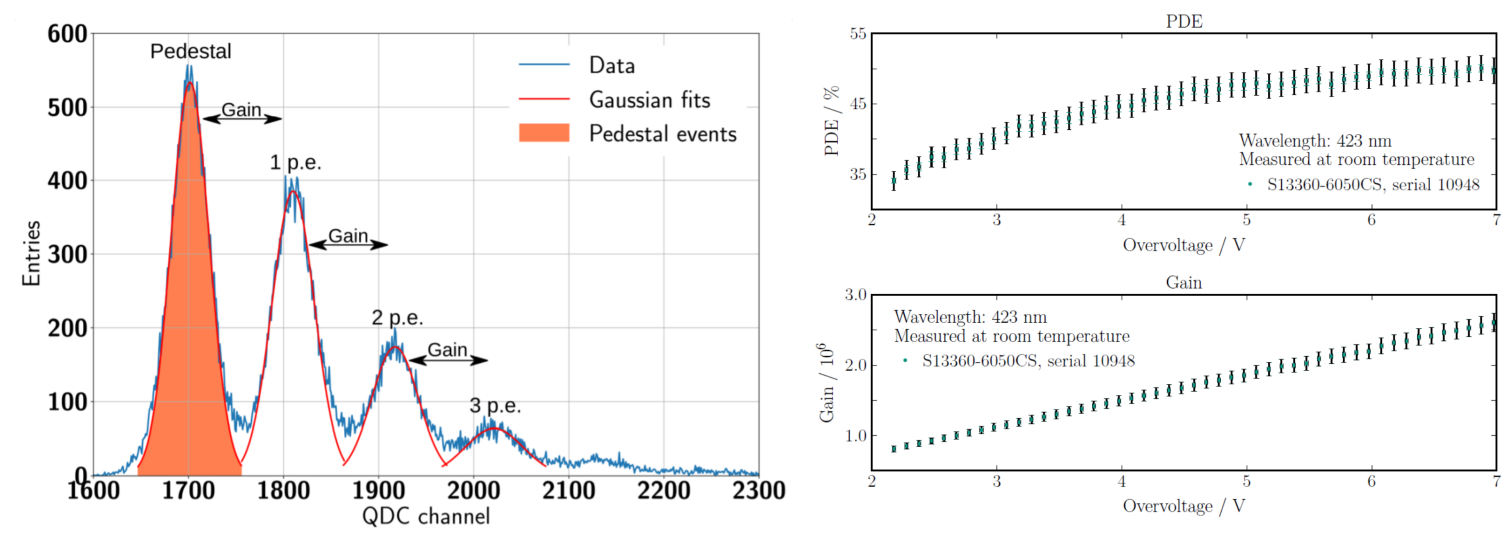

Figure 4: Left: Example of a charge spectrum recorded with one channel of a Hamamatsu SiPM array S13361-3050AS-08, obtained with the Single Photon Calibration Stand at KIT (SPOCK). The red lined Gaussian fits result in the PDE and the Gain of the SiPM (right)

\section{Example application: The IceCube scintillator surface enhancement}

Here we discuss one specific application of SiPMs and the pertinence of the calibration of these devices. In order to improve the performance of the IceCube surface array IceTop, it is foreseen 
to enhance IceTop by an array of scintillator detectors [18]. These scintillators, shown in Figure 5, are readout by a $6 \times 6 \mathrm{~mm}^{2} \mathrm{SiPMs}^{1}$ and IceTAXI [19]. The photons generated by interaction of charged particles in the scintillator material are collected by wavelength shifting optical fibers and transmitted to the SiPM. In the antarctic summer 2017/18 two prototype stations with 7 detectors each were deployed at the South Pole.

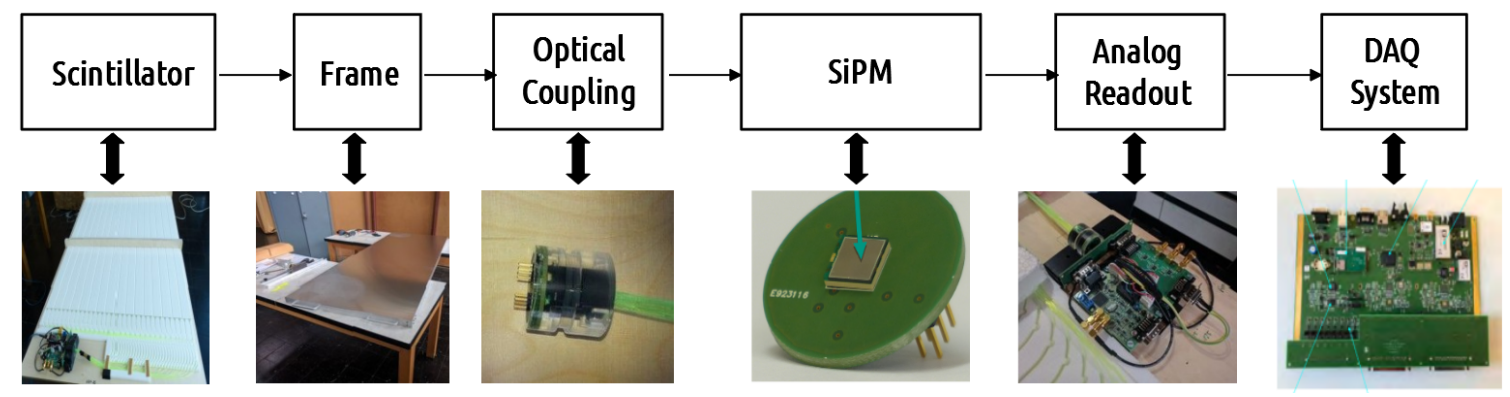

Figure 5: Working principle of an IceCube scintillation detector with SiPM readout. The collected light is transmitted via wavelength shifting optical fibers to the SiPM. Via an analogue double-ended transmission the signal is transferred to the central DAQ, IceTAXI. The gain of the SiPM is controlled via a bias voltagetemperature control loop in order to keep the whole scintillation detector array uniform. The temperature sensor is placed on the backside of the SiPM breakout board.

All SiPMs are characterized via the above described calibration scheme before installation in the detectors to set the optimum operation parameters at the detector electronics. The calibration values are then also used for the interpretation of the detector signals after deployment. Figure 6 shows some of the performed measurements of the characteristics of the deployed SiPM readout of the IceCube scintillation detectors. The measured electrical signal only depend on the charge deposit in the detector, so the amount of measured photo-electrons was determined. In addition, a result of a gain-temperature control loop is shown in Figure 6.

Since SiPMs are solid-state devices, the performance and characteristics, like the gain, depend on the ambient temperature. To ensure a uniform detector array at the South Pole, where the
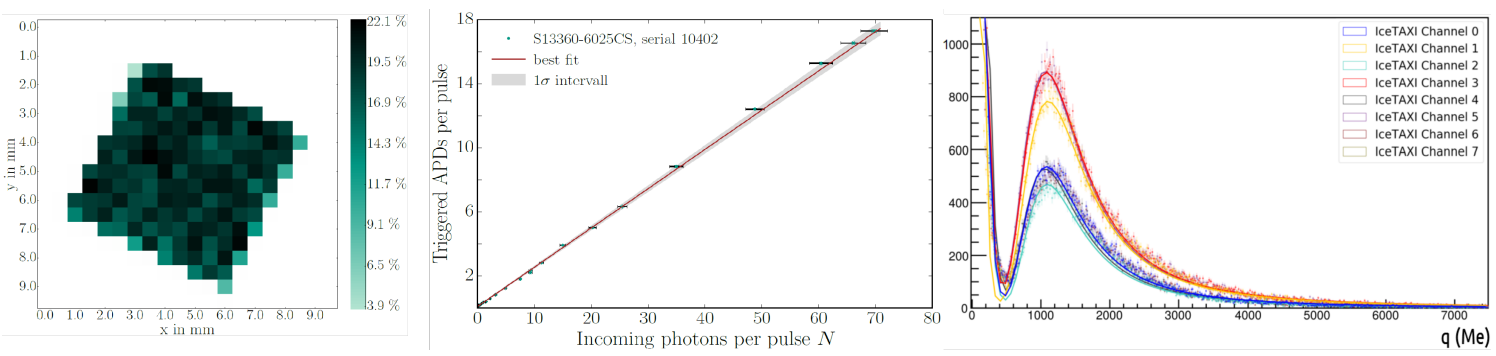

Figure 6: Left: Screened photosensitive area for obtaining the uniformity of the PDE over the whole SiPM. Center: Incoming photons vs. triggered avalanche photo diodes (APDs) of a SiPM, measured to ensure a linear behaviour of the signal output of the photosensor. Right: Charge histograms of the deployed scintillation detectors with enabled temperature control loop. The MIP peak is clearly visible. The amount of entries in the individual IceTAXI channels differs due to the varying snow coverage of the detectors.

\footnotetext{
${ }^{1}$ Hamamatsu 13360-6025PE
} 
temperature fluctuates between $T=[-80 ;-15]^{\circ} \mathrm{C}$ all single detectors are gain stabilized by a control loop realized through adjustment of the SiPM bias voltage. The gain at $T=25^{\circ} \mathrm{C}$ and the temperature behaviour of the SiPMs were measured in the laboratory before the deployment.

\section{Conclusions}

SENSE provides a platform, where experts on photosensors create a common voice towards industry. Therefore, many institutes working in the LLL development area were convinced to work together in this project. SENSE has now reached a new level of publicity, education and training, co-operation in sensor testing, and the preparation of a roadmap which allows us to prepare a final roadmap in which directions of future developments of LLL sensors are recommended to industry and potential customers.

Acknowledgement This project received funding from the European Unions Horizon 2020 research and innovation program under grant agreement no. 713171.

\section{References}

[1] A. Nagai et al. [SENSE Consortium], Nucl. Instrum. Meth. A 912 (2018) 182

[2] A. Nagai et al. [SENSE Consortium], arXiv:1810.02275 [physics.ins-det].

[3] G. Bonanno et al. [SENSE Consortium], J. Phys. Conf. Ser. 1181 (2019) no.1, 012082.

[4] M. Actis et al. [CTA Consortium], Exper. Astron. 32 (2011) 193

[5] P. Bagley et al. [KM3NeT Collaboration], Technical Design Report for a Deep-Sea Research Infrastructure in the Mediterranean Sea Incorporating a Very Large Volume Neutrino Telescope,"

[6] K. Abe et al. [T2K Collaboration], Nucl. Instrum. Meth. A 659 (2011) 106

[7] P. Buzhan et al., Nucl. Instrum. Meth. A 504 (2003) 48.

[8] M. Agostini et al. [GERDA Collaboration], Eur. Phys. J. C 78 (2018) no.5, 388

[9] H. Anderhub et al., JINST 8 (2013) P06008

[10] J. Aleksić et al. [MAGIO Collaboration], Astropart. Phys. 72 (2016) 61

[11] V. Scotti et al. [JEM-EUSO Collaboration], Nucl. Instrum. Meth. A 824 (2016) 655.

[12] T. Bretz, J. Auffenberg, T. Hebbeker, L. Middendorf, T. Niggemann, C. Peters and J. Schumacher, PoS ICRC 2015 (2016) 649.

[13] A. Wright [DarkSide Collaboration], arXiv:1109.2979 [physics.ins-det].

[14] J. Aalbers et al. [DARWIN Collaboration], JCAP 1611 (2016) 017

[15] R. Bähre et al., JINST 8 (2013) T09001

[16] G. Angloher et al., Astropart. Phys. 31 (2009) 270

[17] M. Renschler, W. Painter, F. Bisconti, A. Haungs, T. Huber, M. Karus, H. Schieler and A. Weindl, Nucl. Instrum. Meth. A 888 (2018) 257

[18] S. Kunwar et al. [IceCube-Gen2 Collaboration], PoS ICRC 2017 (2018) 401.

[19] T. Karg, A. Haungs, M. Kleifges, R. Nahnhauer and K.-H. Sulanke, arXiv:1410.4685 [astro-ph.IM]. 